\title{
Estudio sobre las dinámicas familiares de los adolescentes infractores del programa AIMAR del Municipio de Envigado, durante el año $2016^{1}$.
}

\author{
Study about the family dynamics of adolescent law offenders from \\ the AIMAR program of the municipality of Envigado during the 2016
}

\section{Estudo sobre a dinâmica familiar de adolescentes infratores do município de programa Envigado AIMAR durante o ano de 2016}

\author{
Eliana Andrea Cardona Loaiza ${ }^{2}$ \\ Nathalie Martínez Martínez ${ }^{3}$ \\ Olena Klimenko ${ }^{4}$
}

Recibido: 10.11.2016 - Arbitrado: 05.12.2016 - Aprobado: 08.02.2017

\begin{abstract}
Resumen
La presente investigación se orientó a indagar por las algunas características de las dinámicas familiares de los adolescentes infractores del programa AIMAR (atención integral al menor en alto riesgo) del Municipio de Envigado, durante el año 2016. Se utilizó la metodología mixta, nivel descriptivo. Se aplicó dos tipos de instrumentos: entrevista a profundidad y el cuestionario "Como es su familia", diseñado y validado por la Fundacion W.K. Kellogg, la muestra se conformó por 15 familias de los adolescentes entre 14 y 18 años, que se encuentran actualmente en la atención en el programa. Se indagaron las categorías de: comunicación, cohesión familiar, autoridad, afrontamiento de problemas, rutinas familiares, valores y satisfacción con la vida. Los resultados mostraron presencia de dificultades en la comunicación y cohesión familiar, se evidencio, también, que en la mayoría de las familias, la figura paterna se encuentra ausente física o simbólicamente, promoviendo una mayor vinculación al referente materno.
\end{abstract}

Palabras claves: adolescente, familia, dinámicas familiares, menor infractor.

\footnotetext{
${ }^{1}$ Articulo presenta resultados de la investigación llevada a cabo para optar el título de psicólogo, Facultad de Ciencias Sociales, programa de psicología, Institución Universitaria de Envigado, 2016.

${ }^{2}$ Psicóloga, Institución Universitaria de Envigado, elianaa17@hotmail.com

3 Psicóloga, Institución Universitaria de Envigado, natimartinez3007@ hotmail.com

${ }^{4}$ Psicóloga, Universidad estatal Lomonosov, Moscú; Magister en Ciencias Sociales, Universidad de Antioquia; Doctoranda en psicopedagogía, Universidad Católica de Argentina; Docente tiempo completo institución Universitaria de Envigado, Grupo PAYS, eklimenco@correo.iue.edu.co

Citación del artículo: Cardona, E., Martínez, N., Klimenko, O. (2017). Estudio sobre las dinámicas familiares de los adolescentes infractores del programa AIMAR del Municipio de Envigado, durante el año 2016. Revista Katharsis, N 23, enero-junio 2017, pp. 34-59, Disponible en http://revistas.iue.edu.co/index.php/katharsis
} 


\begin{abstract}
This research was oriented to explore some characteristics of the family dynamics of offending adolescents in AIMAR (integral care for the child at high risk) program of the municipality of Envigado, during the year 2016. It used the mixed metodology and descriptive level. Was applied two types of instruments: depth interview and the questionnaire "as you family", designed and validated by the Fundación W.K. Kellogg. The sample is formed by 15 families of adolescents between 14 and 18 years, who are currently in attendance in the program. There was inquired the categories of: communication, family cohesion, authority, coping with problems, family routines, values and life satisfaction. The results showed presence of difficulties in communication and family cohesion, and also was evident, that in the majority of families, the father figure is absent physically or symbolically, that promoting a greater link to the maternal reference.
\end{abstract}

Key words: family, adolescent, juvenile offenders, dynamic family.

\title{
Resumo
}

Esta pesquisa foi orientada para explorar algumas características da dinâmica familiar de ofender adolescentes em programa AIMAR (atenção integral para a criança de alto risco) do município de Envigado, durante o ano de 2016. Foi utilizada a metodologia comum, nível descritivo. Aplicados dois tipos de instrumentos: entrevista com profundidade e o questionário "como a sua família", concebidos e validados pela Fundación W.K. Kellogg, a amostra foi formada por 15 famílias de adolescentes entre 14 e 18 anos, que estão atualmente presentes no programa. Perguntaram as categorias de: comunicação, a coesão familiar, a autoridade, lidar com problemas, rotinas familiares, valores e satisfação de vida. Os resultados mostraram a presença de dificuldades de comunicação e coesão familiar, evidencio, também, que a figura do pai é ausente fisicamente ou simbolicamente, na maioria das famílias, promovendo uma maior ligação à referência materna.

Palavras-chave: família, adolescentes, jovens infratores, família dinámica.

\section{Introducción}

La familia constituye un núcleo básico de la sociedad humana que permite al niño establecer sus primeras relaciones con las personas significativas de su vida y determina, en cierto grado, la formación que recibirá este niño, aprendizaje social que tendrá y experiencias

Citación del artículo: Cardona, E., Martínez, N., Klimenko, O. (2017). Estudio sobre las dinámicas familiares de los adolescentes infractores del programa AIMAR del Municipio de Envigado, durante el año 2016. Revista Katharsis, N 23, enero-junio 2017, pp. 34-59, Disponible en http://revistas.iue.edu.co/index.php/katharsis 
claves por los cuales pasará, influenciando de manera significativa su futura relación consigo mismo y con los demás.

Formar de manera asertiva e integral a los niños desde la familia y la escuela, es de vital importancia para prepararlos a enfrentar las problemáticas sociales contemporáneas. Ayudarlos a fortalecer su ser individual, emocional y social, podrá formar en ellos nuevas herramientas para crear sociedades diferentes y familias más comunicadas (Palacio Valencia, 2010).

Viveros \& Vergara (2006) afirman que desde la familia se preparan herramientas para enfrentar el mundo social de diferentes formas, allí se encargan de formar en valores, normas, manejo de emociones, que contribuyen al fortalecimiento de las habilidades y las relaciones sociales, la autoestima, las expresiones afectivas y las relaciones entre los miembros de la familia, y a esto lo denomina "supervivencia afectiva y social", pues desde que se crean medios efectivos para una adecuada inserción social, el niño, un futuro adulto, sobrevivirá emocionalmente en una cultura que ataca constantemente y crecerá con las herramientas suficientes para enfrentar las dificultades que emerjan en la cultura.

Por otro lado, la desintegración familiar, que tiene su origen en diferentes causas, permite que otras problemáticas empiecen a desarrollarse dentro del núcleo familiar. Un ejemplo de ello es la violencia, que históricamente se presenta en diferentes escenarios, impartiendo creencias, comportamientos y pensamientos que se instauran en las familias, naturalizando comportamientos que pueden ser dañinos y que se convierten en nuevas problemáticas sociales (Gaxiola \& Frías, 2008).

Los ambientes familiares que presentan problemáticas en sus dinámicas pueden generar incidencia en el comportamiento de los niños y adolescentes produciendo comportamientos que perjudican su desempeño social (Chassin et al, 2004; Oliva et al, 2008).

Según Pons Diez (1998), el sistema familiar desempeña un papel fundamental en la formación de los niños y adolescentes, incidiendo en la aparición de diferentes conductas desadaptativas como consumo de drogas, actos que infringen la ley, etc. Los contextos sociales como medios de comunicación, grupo de iguales, escuela, etc., pasan por "el tamiz de la familia, que puede tanto amplificar como disminuir sus efectos e influencias, sean estos positivos o negativos" (p. 252).

Los investigadores resaltan la incidencia que tienen las características de las dinámicas familiares en diferentes aspectos de la vida y comportamiento de los niños y adolescentes, como, por ejemplo: buen acompañamiento familiar y adecuada comunicación en familia incide en el éxito escolar de los niños (López et al, 2005; Balarin \& Cueto, 2008; Gutiérrez Cárdenas, 2014); una positiva percepción de la funcionalidad familiar en los adolescentes esta relacionada con un menor riesgo biopsicosocial (Ulate-Gómez, 2013); los factores como falta de comunicación y alejamiento afectivo en las familias se relacionan con el consumo y el temor de los adolescentes para comunicarse con sus padres (Cobos, 2008); el estilo 
educativo basado en el afecto y comunicación asertiva, al parecer, permite contrarrestar la influencia de ambientes sociales negativos en los adolescentes y prevenir que se involucren en las conductas delictivas (Fuentes et al, 2015; Mosqueda-Díaz \& Ferriani, 2011; Amezcua et al, 2016); igualmente, una menor cohesión familiar, ausencia de lazos afectivo emocionales y desunión entre sus integrantes, actúa como un factor que propicia el comportamiento delictivo y consumo de sustancias psicoactivos en los adolescentes (Martínez et al, 2014; Arias, 2013).

Cuando se trata de estudiar la familia, es importante orientar atención al estudio de dinámicas familiares que permiten identificar aspectos relacionados con la comunicación, cohesión familiar, ejercicio de autoridad, procesos de afrontamiento de problemas, aspecto de valores y satisfacción con la vida en las familias.

Franco (2004, citado en Gallego, 2011) señala que todo grupo familiar tiene su dinámica interna y externa, la primera se refiere al contexto familiar y a las interacciones que se crean; y la dinámica externa, tiene que ver a la relación de la familia en el entorno social y como está la afecta, para que surja o se estanque.

De la misma forma, Oliveira, Eternod \& López (1999), Torres, Ortega, Garrido \& Reyes (2008), González (2000) y Palacios \& Sánchez (1996, citados en Gallego, 2011) subrayan que la dinámica familiar es el tejido de relaciones y vínculos atravesados por la colaboración, intercambio, poder y conflicto que se genera entre los miembros de la familiapadre, madre e hijos- al interior de ésta, de acuerdo a la distribución de responsabilidades en el hogar, la participación y la toma de decisiones.

Cuando se atiende a la población infantil o adolescente, es imprescindible estudiar también la familia y sus dinámicas para una mayor comprensión de causas y posibles focos de abordaje para las dificultades que presenta un niño o adolescente.

Más aun, cuando se trabaja con una población de adolescentes que presenta diferentes problemáticas, como por ejemplo, menores infractores con historial de consumo de sustancias, es de gran importancia orientar atención a las familias de estos, con el fin de poder determinar si sus familias cumplen con "las principales funciones de la familia como proveer alimentación, hábitat, salud, protección, afecto y seguridad” y, además, cuentan “con estrategias y formas de comunicación, conocimientos, costumbres, valores, sentimientos, normas de comportamiento y de relación con los demás" (Parada Navas, 2010, p.18) necesarios para poder ayudar a los adolescentes a superar sus dificultades.

El presente estudio se realizó en la población de los adolescentes menores infractores pertenecientes al Programa AIMAR del Municipio de Envigado, quienes cuentan con múltiples problemas, iniciando por el consumo de sustancias psicoactivas y terminando con la infracción y violación a la ley. Se orientó el interés del estudio a las dinámicas familiares en los cuales participan estos jóvenes en sus familias de origen, con el fin de mirar si existen algunas dinámicas disfuncionales, que podrían ser intervenidas desde los programas de salud

Citación del artículo: Cardona, E., Martínez, N., Klimenko, O. (2017). Estudio sobre las dinámicas familiares de los adolescentes infractores del programa AIMAR del Municipio de Envigado, durante el año 2016. Revista Katharsis, N 23, enero-junio 2017, pp. 34-59, Disponible en http://revistas.iue.edu.co/index.php/katharsis 
mental del municipio, al igual como identificar aspectos positivos presentes en relaciones familiares que podrían fortalecerse y servir como factores protectores en los procesos de atención psicosociales llevados a cabo con esta población.

\section{Metodología}

El estudio fue de enfoque mixto y nivel descriptivo.

\section{Participantes}

La población elegida, consta de 15 familias, de las que hacen parte los adolescentes entendidos en el programa de Atención Integral al Menor en Alto Riesgo (AIMAR) de la secretaria de bienestar social del municipio de Envigado.

La muestra poblacional para la realización de este proyecto, fue tomada a conveniencia, contando con los 16 adolescentes entre los 14 y 18 años de edad y sus respectivas familias que se encuentran atendidos en un proceso terapéutico individual en el programa.

\section{Instrumentos}

El instrumento utilizado fue un cuestionario denominado "Como es tu familia", diseñado por la fundación W.K. Kellogg en el 1996 y avalado por la OPS (Organización Panamericana de la Salud), que evalúa elementos de adaptabilidad y vulnerabilidad familiar a través de los cuestionarios que constan de 25 peguntas para los adolescentes y 30 para los padres. De todo el cuestionario, en este trabajo solo se presentan las categorías de: comunicación, cohesión familiar, ejercicio de autoridad en familia, rutinas familiares, afrontamiento de problemas, satisfacción con la vida.

Adicionalmente, se realizaron las entrevistas a profundidad con los adolescentes, que permitieron proporcionar información de orden cualitativa, adicional al cuestionario, cuyo análisis se contrasto y se complementó con la información obtenida en el cuestionario según las categorías estudiadas.

\section{Aspectos éticos}

Esta investigación se soportó en el código deontológico del psicólogo y la resolución № 8430 de 1993, por la cual se establecen las normas científicas y técnicas y administrativas para la investigación en salud, se aplicó el consentimiento informado, firmado por los padres de familia.

\section{Resultados y discusión}

Citación del artículo: Cardona, E., Martínez, N., Klimenko, O. (2017). Estudio sobre las dinámicas familiares de los adolescentes infractores del programa AIMAR del Municipio de Envigado, durante el año 2016. Revista Katharsis, N 23, enero-junio 2017, pp. 34-59, Disponible en http://revistas.iue.edu.co/index.php/katharsis 


\section{Comunicación en familias}

Los datos muestran que hay una marcada diferencia como los adolescentes dialogan con su padre y madre, en donde el 53\% afirmaron estar satisfechos por la forma en que conversan con su madre y en un $40 \%$ afirma lo contrario en cuanto a su padre o figura paterna. Por su parte, la familia (padre o madre o quien representara estas figuras) argumentaron estar satisfechos solo en un $20 \%$.

En cuanto a la expresión de los sentimientos dentro de la familia, los adolescentes manifiestan dificultades para expresarse tanto a padres como a madres. El 28\% indican que es fácil expresarle todos sus sentimientos a la madre en algunas ocasiones y la gran mayoría enuncia que al padre nunca los expresan.

Solo $33 \%$ de los adolescentes participantes expresaron poder hablar con sus padres sobre las dificultades que atraviesen, y la figura de este contacto en estos momentos de dificultad fue la madre, y $40 \%$ expresaron que nunca podía hacerlo con su padre.

Por su parte, padre y/o madre manifiestan poder expresar fácilmente sus sentimientos a sus hijos solo con un $47 \%$ y un $40 \%$ dicen que casi siempre expresan lo que sienten a sus hijos.

Lo anterior indica que, en general, en las familias participantes se presentan dificultades en la confianza entre sus miembros para expresarles lo que sienten y en cuanto a las dificultades que atraviesan, situación que podría ser normal para los adolescentes debido a su etapa evolutiva en la que se encuentran, sin embargo, esto se convierte en una problemática si dentro del núcleo familiar no se fomenta la comunicación y el dialogo constante. Según Domínguez \& Rodríguez (2003), la expresión y comprensión de los sentimientos entre los miembros de una familia es importante para crear el clima de seguridad en la familia. A su vez, este clima emocional se relaciona con la formación de niños y adolescentes permitiendo fomentar la confianza en ellos mismos, su autoestima y actúa como un factor protector para el consumo de sustancias (Medina \& Carvalho, 2010; Pichardo, 1998).

A partir de las entrevistas también se evidencia que en la mayoría de las familias estudiadas, se genera un tipo de comunicación inadecuada, tanto de padres hacia hijos, como viceversa. Los adolescentes comentan que tienen poca comunicación con los padres y otros miembros de la familia:

"yo no me meto con nadie y ellos no se meten conmigo. Yo vivo encerrado en mi cuarto cuando estoy, pero por lo general no me gusta estar ahí"

"Soy muy callada y trato de no poner problema, no me gusta contar mis cosas. Con mi papá casi no podemos hablar"

"Si hablamos es siempre para pelear" "ella (mamá) siempre regaña y me trata mal"

Citación del artículo: Cardona, E., Martínez, N., Klimenko, O. (2017). Estudio sobre las dinámicas familiares de los adolescentes infractores del programa AIMAR del Municipio de Envigado, durante el año 2016. Revista Katharsis, N 23, enero-junio 2017, pp. 34-59, Disponible en http://revistas.iue.edu.co/index.php/katharsis 
"Yo con mi mama nunca puedo hablar, ella todo el tiempo se la pasa trabajando y no más, y cuando llega a la casa es como aburrida o malgenio"

Igualmente, expresan que, a pesar de contar con alguien en la familia con quien pueden compartir sus cosas (generalmente es la figura materna), existe una situación de conflicto al interior de las familias, debido a una mala comunicación y la incapacidad de establecer un dialogo de convivencia:

"Mi mamá y yo si hablamos mucho, ella trata de entenderme mucho, pero mi familia es un caos, pelean por todo, gritan, se estresa todo el mundo"

La mayoría de los adolescentes tiene una relación de comunicación más cercana, aunque difícil, con la figura materna, el padre participa muy poco o casi nada en la comunicación con estos:

"Algunas veces podemos hablar, sin embargo, por lo general solo alega, ella no escucha y con mi papá la verdad no me importa, el allá y yo acá."

"La mayoría de las veces cada que hablo con mi mama es para pelear, ella todo el tiempo está alegando y se queja por todo, promete muchas cosas pero no cumple nada. De mi papa casi no sé nada aunque hace poquito me dijo que me iba a empezar a mandar plata”

A su vez, los padres de familia, se quejan de la postura reservada de sus hijos, y, al mismo tiempo, comunican que ellos tampoco se comunican con sus hijos de forma abierta y constante:

"Nunca expresa nada de lo que le pasa en la casa, uno siempre se entera por otro lado de todo"

"yo también hablo poco, no me gusta que se enteren cuando tengo alguna dificultad"

"En nuestra casa solo algunas veces hablamos entre nosotros para comunicarnos o expresarnos cosas, somos muy callados, me gustaría tener más comunicación"

"Ese muchacho es muy callado, casi no habla, ahí responde como por responder y ya"

"Casi no hablamos, son muy pocas las veces que compartimos algo juntos, Yo no sirvo para rogarle a nadie, Ese muchacho es muy callado y nunca habla"

"Mi hija no habla nunca y yo tampoco le pregunto, esa niña es muy desobediente y quiere hacer lo que le da la gana conmigo, sino yo ya no le voy a rogar" 
Los padres también expresan intenciones comunicativas, pero, al mismo tiempo, expresan dificultades para encontrar formas de acercamiento a sus hijos:

"Yo trato siempre de comunicarme con mi hijo, decirle las cosas, pero es que es muy terco, no escucha"

"Ella casi no habla, es muy callada y a uno le toca rogarle para que diga alguna cosa, Yo trato de acercarme pero ella es muy reservada con sus cosas"

"Él está muy perdido, ya el solo quiere calle y no más, yo solo quiero entregar esa custodia no lo aguanto, está muy grosero"

Lo anterior muestra presencia de problemáticas a nivel comunicativo en familias, tanto por parte de los adolescentes, como por parte de los padres de familia.

Los autores resaltan la importancia de factores familiares, y entre estos, el aspecto de calidad de comunicación familiar, en el consumo de sustancias psicoactivas y conductas transgresoras en los adolescentes. Para Amezcua et al. (2016) la ambivalencia de la comunicación provoca factores de riesgo que desencadena conductas antisociales o delincuenciales de los hijos. Fantin \& Garcia (2011) indican que los problemas en comunicación con los padres aumentan la posibilidad de consumo de sustancias en los adolescentes. Igualmente, el conflicto familiar a nivel general de familia, la ausencia de dialogo entre sus miembros y tendencia a resolver los conflictos mediante peleas, lo cual igualmente, fue manifestado en las entrevistas, es un factor vinculado con el consumo de drogas en los adolescentes (Sanz et al, 2004).

Como se puede ver la categoría de comunicación mostro deficiencias notorias presentes en las familias de los menores infractores, lo cual actúa como un factor de riesgo para su proceso de rehabilitación en cuanto al consumo de sustancias psicoactivas.

\section{Cohesión familiar}

En cuanto a la cohesión familiar, igualmente, se encontraron falencias en la mayoría de las familias participantes. $47 \%$ de los adolescentes informan que les gusta pasar el tiempo libre con su familia, mientras para los padres es una preferencia solo para un $27 \%$.

Un $73 \%$ de los adolescentes reportan que no expresan fácilmente lo que desean con sus familiares y los padres tampoco lo hacen en un 67\%. En cuanto a pedir ayuda unos a otros, solo un $33 \%$ de los adolescentes encuestados argumentan que con frecuencia lo hacen, al igual como los padres, con el mismo porcentaje.

Por otro lado, según los adolescentes, sus ideas son tenidas en cuenta en la solución de los problemas en familia solo en un $14 \%$ y un $47 \%$ de los padres expresaron que los hijos participan en las decisiones familiares. Solo un $7 \%$ de los adolescentes y los padres, por igual, respondieron que la familia se une para resolver asuntos de importancia familiar.

Citación del artículo: Cardona, E., Martínez, N., Klimenko, O. (2017). Estudio sobre las dinámicas familiares de los adolescentes infractores del programa AIMAR del Municipio de Envigado, durante el año 2016. Revista Katharsis, N 23, enero-junio 2017, pp. 34-59, Disponible en http://revistas.iue.edu.co/index.php/katharsis 
Lo anterior muestra un bajo nivel de cohesión en las familias, donde se comparta poco tiempo y espacios en conjunto entre sus miembros, mostrando una tendencia al individualismo por parte de cada uno.

Otra de las partes que determina la cohesión familiar es la codistribución de las responsabilidades del hogar, donde los adolescentes indicaron solo en un 33\% que las responsabilidades son compartidas y $40 \%$ de los padres dieron la misma respuesta.

La cohesion familiar también está relacionada con el grado de contacto con las familias de origen, abuelos, tíos, primos, etc. En este sentido solo 33\% tanto para los adolescentes, como para los padres, los participantes indicaron que comparten eventos con parientes. Sin embargo, a pesar de un porcentaje bajo de contacto directo con los parientes, se encuentra una relativamente buena satisfacción frente a la relación con sus parientes cercanos y lejanos. $50 \%$ para los adolescentes y un $40 \%$ para los padres.

En las entrevistas igualmente, se evidencia el hecho de un bajo nivel de contacto directo entre los miembros de las familias, mostrando que la mayoría llevan vidas individuales, solo compartiendo el espacio vital de una casa, sin contar realmente con un grado suficiente de cohesión, la cual permite crear lazos afectivos entre sus miembros.

Estas afirmaciones se pueden observar por parte de los adolescentes:

"En la situación en la que estoy yo solo puedo ir a esa casa a dormir, no pasamos ningún tiempo juntos y hasta me dicen que si no llevo comida no me dejan entrar"

"Yo siempre como con todos pero casi no nos hablamos, ellos son queridos pero no se meten conmigo, solo cuando yo necesito algo"

"Nosotros no compartimos nada juntos. Yo como en mi habitación y no me gusta que me pregunten por la vida que llevo, que no se metan"

"no me la llevo bien con mi prima que vive en la casa, no hablo con ella, tengo un medio hermano y de vez en cuando salimos pero no más, y con mi mamá que la veo todos los días, normal"

"Somos una familia normal, no nos mantenemos juntos y casi nunca hablamos de los problemas"

"No comparto con mi mamá ni con mis hermanos, vivo con mi abuela, mi abuelo y una prima, tampoco comparto mucho tiempo con ellos solo lo que hagamos en la casa"

Los padres también expresan poco contacto con los hijos, exponiendo la cuestión del trabajo como una justificación de no contar con tiempo suficiente:

"Yo algunas veces lo llamo para que comamos algo juntos, pero ese niño no quiere nada que tenga que ver conmigo el solo quiere calle 
"Pasamos en la casa la mayoría del tiempo, aunque casi no hablamos cada uno es muy aparte

"Todos en la casa son como por su lado, aunque mis hermanos y mis papas adoran a mi hijo, claro porque nunca se enteran de lo que pasa con él, ni por mi lado y por el de él menos"

"Yo no tengo tiempo de hacer nada diferente a trabajar y sobre todo porque mi hija tampoco casi no ayuda en nada, Ella solo quiere irse con esas amigas de por ahi" busque"

"A mí no me queda tiempo, pero él sabe que cuando me necesite, que me

También los padres reportan la presencia de pocos vínculos con la familia extensa, incluyendo los vínculos con sus familias de procedencia, tíos, primos, etc., sin embargo, al igual como se mostró en el cuestionario, a pesar de poco contacto que tienen los padres y adolescentes con otros miembros de familias extensas, reportan tener buenas relaciones con ellos:

"Vivimos solos acá, mi familia nos ayuda económicamente pero no nos visitamos a menudo"

"Insisto en que si ellos no me hacen participe de sus actividades, yo no les puedo rogar, voy hasta donde me lo permitan, sin embargo, yo con mi familia tengo buenas relaciones."

"No somos muy unidos con los parientes que viven fuera de la casa, pero no nos llevamos mal con nadie"

Algunos adolescentes expresan un mayor grado de acercamiento con otros miembros de familia que no entran directamente en el núcleo familiar primario, tales como abuelos, tíos, o también, con personas diferentes, como madrinas, por ejemplo:

"Mi mama no tiene tiempo para nada y casi no compartimos cosas, me dice que se mantiene muy cansada, yo prefiero pedirle ayuda a mi madrina, ella está más pendiente de mí"

"mis tíos tías y abuelos me quieren mucho"

En general, se denota la tendencia hacia un bajo nivel de contacto entre los miembros de familias, tanto al interior de estas, como con otros parientes por fuera del núcleo familiar, mostrando intensión de poner distancia con el fin de permanecer en su espacio personal tanto al interior de las familias, como hacia afuera, procurando, a su vez, que las dificultades que ocurren al interior del hogar sean solucionadas de manera individual y privada.

Los datos obtenidos, tanto en esta categoría, como en la anterior, confirman los hallazgos de Ortiz et al. (2006), quien afirma que "en las familias de consumidores de

Citación del artículo: Cardona, E., Martínez, N., Klimenko, O. (2017). Estudio sobre las dinámicas familiares de los adolescentes infractores del programa AIMAR del Municipio de Envigado, durante el año 2016. Revista Katharsis, N 23, enero-junio 2017, pp. 34-59, Disponible en http://revistas.iue.edu.co/index.php/katharsis 
sustancias que delinquen, existe menor cohesión familiar, indicando ausencia de lazos afectivos emocionales, y desunión entre sus integrantes. Así mismo, en estas familias existe mayor conflicto caracterizado por discusiones frecuentes y énfasis en aspectos negativos de la interacción" (p. 116).

Según algunos autores (Basto et al., 1978; Cañavera, 1988; Watzlawick, 1974; Ausloss, 1983; Valverde, 1991; Fuerte \& Maya, 2005) la baja cohesión familiar esta relacionada con las conductas de riesgo y el consumo de sustancias en los hijos.

En este aspecto es las familias participantes la baja cohesión familiar, emerge como un factor de riesgo para los adolescentes que se encuentran en el programa, tanto para el consumo de sustancias que presentan, como para las conductas delincuentes.

\section{Ejercicio de autoridad}

Para el 55\% de los adolescentes la autoridad en la familia se centra en la figura materna. Este hecho de predominancia de figura materna como figura de autoridad en la casa se refleja también en las expresiones de los adolescentes:

"Mi mamá me deja tareas para hacer en la casa y aunque son muchas yo las hago"

"En mi casa, mi mamá es la que regaña" "yo siempre le hago caso y respeto"

"Mi mamá manda por todo, me jode mucho"

"En mi casa manda mi mamá a veces, yo sé cómo hacer para que me deje hacer lo que quiero y no moleste"

Sin embargo, en las respuestas de los adolescentes también se puede observar un alto grado de irrelevancia e indiferencia frente al ejercicio de autoridad de los adultos:

"Pues se supone que mi papá, pero a mí me da igual, cada quien en lo suyo"

"Mi mamá trata de tener autoridad pero yo siempre hago lo que quiero" "es que es demasiado regañona"

"Yo creo que así mi mamá diga que no, yo creo que cada uno hace lo que quiere porque así ella diga que no, uno siempre termina haciendo lo que uno quiere"

"Mi mama me dice que le haga caso a ella, pero al final siempre dice mentiras, yo que caso le voy a hacer si ni siquiera puede ponerme cuidado"

"Mi mama es la que manda, yo tengo que aceptar que muchas veces no le hago caso y por eso es que tenemos problemas pero ella tiene que entender que yo soy joven y tengo que vivir cosas"

Citación del artículo: Cardona, E., Martínez, N., Klimenko, O. (2017). Estudio sobre las dinámicas familiares de los adolescentes infractores del programa AIMAR del Municipio de Envigado, durante el año 2016. Revista Katharsis, N 23, enero-junio 2017, pp. 34-59, Disponible en http://revistas.iue.edu.co/index.php/katharsis 
Igualmente, se observa que un $29 \%$ en las familias el referente de autoridad es representado por una persona diferente a la madre y/o padre, siendo abuelos, tíos, primos, entre otros, quienes representan figuras de autoridad para los adolescentes. Lo anterior muestra que el ejercicio de la autoridad en muchas familias participantes está mediado no por el hecho de ser padre o madre, sino por la figura de importancia quien asume el rol de autoridad en la familia, aunque no sea un miembro directo de esta.

"Mi mamá es la que manda cuando vivía con ella, pero acá es mi abuelo. A él le obedezco casi siempre"

"Mi tía es la que manda ahora que vive con nosotros, es la que está pendiente y me pone los horarios para entrar a la casa"

En las respuestas de los padres se percibe una impotencia frente al ejercicio de autoridad en la familia, mostrando un fracaso en el intento de establecer normas y reglas de comportamiento tanto al interior como por fuera de la casa:

"No, estos pelaos ya están muy grandes, ya no los manda nadie"

"Atiende a lo que le pedimos pero a veces quiere hacer lo que se le da la gana" "a veces me hace caso otras no"

"La mayoría de mis hijos acatan las ordenes que se les da en la casa, pero en ocasiones hay muchas dificultades con la norma y no puedo ejercer autoridad efectivamente"

"En la casa cada quien hace lo que quiere, mi hijo dice que me hace caso pero si algo no le gusta no dice nada y simplemente hace lo que le parece"

"Soy yo el que de alguna manera dice que no a algunas cosas, pero ellos ya deberían saber que tienen que hacer"

"Yo tengo la autoridad en la casa, aunque mi hijo no hace caso casi nunca a lo que le digo, le encanta ser desafiante"

"No creo que el límite y la autoridad mi hijo lo tenga interiorizado, ese niño es muy grosero y quiere mandarse solo"

"A esa niña no la manda nadie, siempre hace lo que le da la gana pero conmigo no más, yo ya me canse de estar buscándola y trasnochando porque ella no obedece"

"Yo no tengo autoridad sobre él, yo quiero entregar la custodia de él, no se deja mandar de nadie y ya me canse quiero tirar la toalla ya lo perdí"

Igualmente, varias respuestas tanto de los adolescentes, como de los padres demuestran ausencia de un acuerdo intrafamiliar en cuanto al ejercicio de autoridad, lo cual lleva a que el adolescente pueda manipular y desacatar reglas propuestas.

Citación del artículo: Cardona, E., Martínez, N., Klimenko, O. (2017). Estudio sobre las dinámicas familiares de los adolescentes infractores del programa AIMAR del Municipio de Envigado, durante el año 2016. Revista Katharsis, N 23, enero-junio 2017, pp. 34-59, Disponible en http://revistas.iue.edu.co/index.php/katharsis 
"Conmigo, la autoridad la tiene mi mamá, en mi casa eso es un despelote, a veces mi abuela, a veces mi mamá"

"La autoridad en mi casa esta desordenada, pero sobre mi hija trato de ser yo la mayoría de veces aunque a veces tratan de desautorizarme"

Lo anterior denota problemáticas relacionadas con la autoridad, establecimiento y cumplimiento de reglas al interior de las familias participantes.

Según Navarrete \& Ossa (2013), existe una relación entre el mal comportamiento de los hijos, con un grado de permisividad y baja orientación por parte de los padres, bajo control sobre sí mismos, e inseguridad por el hecho de que no poseen las herramientas necesarias para afrontar situaciones de riesgo en las que se puedan ver involucrados; situación que puede evidenciarse en los testimonios de los participantes.

Vásquez (2003) expone que las problemáticas relacionadas con el ejercicio de autoridad en las familias, como ausencia de una clara figura de autoridad al interior de familias, conflictos, y negligencia frente al control ejercido por parte de los padres, actúan como factores de riesgo para la delincuencia juvenil y el consumo de sustancias.

A partir de las anteriores deficiencias encontradas en las familias participantes en relación al ejercicio de autoridad se hace evidente la necesidad de realizar las intervenciones orientadas a mejorar este aspecto de dinámicas de las familias de los menores infractores entrevistados.

\section{Rutinas familiares}

Un $47 \%$ de los adolescentes y $40 \%$ de los padres indicaron que en sus familias no se comparte tiempo en familia mediante realización de diferentes actividades en conjunto. Es un porcentaje significativo, considerando que el hecho de pasar el tiempo libre en familia es un indicador significativo para la construcción de su cohesión y fomento de lazos afectivos entre los miembros.

Este hecho presente en un gran porcentaje de familias se refleja también en las entrevistas:

"Nunca salimos juntos, cada quien hace sus cosas por aparte, mamá trabaja, yo estudio y mi hermanita también"

"En la casa no pasamos mucho tiempo juntos pero cada quien dice lo que piensa"

"En la casa nadie se sienta a hacer cosas juntos, de pronto cuando vamos a comer y eso que la mayoría de veces comen en la habitación"

"Como casi no hay tiempo no programamos pues como para salir, todos tenemos cosas que hacer y yo trabajo todo el día"

"Nosotros no hacemos nada juntos, cada uno está en lo suyo, ni siquiera comen en la mesa."

Citación del artículo: Cardona, E., Martínez, N., Klimenko, O. (2017). Estudio sobre las dinámicas familiares de los adolescentes infractores del programa AIMAR del Municipio de Envigado, durante el año 2016. Revista Katharsis, N 23, enero-junio 2017, pp. 34-59, Disponible en http://revistas.iue.edu.co/index.php/katharsis 
"Todo el tiempo yo estoy trabajando y mi hija estudiando. No me queda tiempo para esas cosas"

"Nunca hacemos nada juntos"

Un porcentaje menor de familias informan, tanto por parte de los adolescentes, como padres, que comparten tiempo junto. Pero lo que se puede ver a partir de sus testimonios, este compartir se limita a ciertas cosas cotidianas, como mercar, comer juntos, celebrar fiestas culturalmente establecidas, visitas familiares o asistencia a citas médicas. Aunque este tipo de cosas son importantes para una familia, también es necesario que las actividades para compartir juntos sean de diversión y libre esparcimiento, cuyo objetivo sea solo de estar juntos y compartir, prestar atención al otro y reforzar la relación entre miembros de la familia, permitiendo de esta forma crear lazos de pertenencia y apoyo.

"El papá se la pasa trabajando. A estos pelaos hay veces es muy difícil cogerlos En familia si nos gusta celebrar fiestas y reuniones."

"Solo la acompaño a hacer mercado, casi nunca nos sentamos en la casa a charlar, nunca salimos, lo único que a veces hacemos juntos es comer"

"Solo en ocasiones planeamos hacer cosas juntos, sin embargo, siempre trato de crear rutinas en la casa como comer juntos"

"Todos los días comemos juntos, pero no salimos cada tanto ni nada"

"De vez en cuando salimos donde familiares, es que casi no hay tiempo por el trabajo"

"Yo siempre salgo solo con mis amigos, con mi mamá solo salgo cuando tengo citas médicas o psicológicas"

Según lo que expresan algunos adolecentes, se puede ver que este tipo de rutinas familiares como comer juntos, realmente representan solo un estar juntos o cerca físicamente, sin embargo, no se da un contacto e intercambio entre las personas participantes, comen juntos pero no hablan entre sí:

"con mi mama comemos juntos pero casi no hablamos, con mi papa no compartimos nada, que pereza"

Un porcentaje aún menor de familias participantes intenta crear algunas rutinas familiares que permiten tener un contacto más cercano a partir de actividades como diversión y juegos:

"Al menos unas vez a las semana jugamos parqués, y comemos en la mesa" "Si, al menos el sábado tratamos de reunirnos para jugar algún juego de mesa. Y por lo general compartimos una de las comidas."

"Yo con mi hija salgo bastante, vamos a cine, a pasear, y en familia solo salimos en ocasiones especiales" 
También se puede notar en las expresiones de los participantes una cierta renuncia a compartir con sus familiares, demostrando un quiebre en las relaciones, tanto por parte de los adolescentes, como por parte de los padres:

"Ellos no quieren compartir nada conmigo y yo no voy a rogarles"

"Yo todo el tiempo estoy en la calle, me despierto temprano barro, trapeo y me abro. Yo no hago nada ni como mi mama ni con mi hermana"

"Yo no hago nada con él, cuando le da la gana se desaparece y no sé nada de el por muchos días, eso ya es muy difícil, yo mientras que no tenga donde quedarme ni modo"

"No todos los días hablamos, pero ya si más que antes, muchas veces los papas si tratan de acercarse más a nosotros solo que uno hay veces es el que no quiere"

"Yo no puedo estar saliendo a toda hora, tengo mucho que hacer, una obligación que llevar a mi madre, a mí nadie me ayuda, todo me toca sola y para acabar de ajustar ella no da sino problemas, yo estoy cansada"

Igualmente, un $47 \%$ de los adolescentes indicaron que sus padres no dedican tiempo a diario para hablar con ellos. Igualmente, se detecta la misma queja proveniente de los padres, que intentan a acercarse a sus hijos pero encuentran la resistencia a compartir y comunicarse por parte de estos.

"Mi mama no tiene tiempo para dedicarme, yo le digo que hagamos cosas juntas pero siempre me dice que está cansada que ahora no. Yo no sé para qué me tuvo"

"A nosotros nos gusta compartir muchas cosa con los hijos, tratamos de integrarnos pero hay veces ellos son más esquivos con eso, pero uno hace el intento"

Los datos encontrados muestran notorias deficiencias en las rutinas familiares de las familias participantes. Esto se relaciona con las problemáticas que se evidencian en la comunicación en las familias, siendo este asunto determinante para un buen clima afectivo dentro de las familias.

Con respecto a las rutinas familiares se observa desde las entrevistas que mientras que los padres responden de forma más positiva a la creación de rutinas dentro de la familia, los adolescentes se muestran más resistentes a realizar actividades recurrentes con sus padres, esto muestra una gran diferencia con respecto a compartir el tiempo libre con la familia, lo cual evidencia una dificultad en el vínculo de padres a hijos que no les permite una constancia en las actividades que puedan realizar juntos, por ello tienen más apertura a compartir tiempo con otras personas de su familia. Las relaciones familiares, son como lo afirma Mosqueda \& Ferriani (2011), percibidas como factores protectores en la que se destaquen actividades que permitan a sus miembros compartir diariamente momentos gratos en familia. Ello ayuda a

Citación del artículo: Cardona, E., Martínez, N., Klimenko, O. (2017). Estudio sobre las dinámicas familiares de los adolescentes infractores del programa AIMAR del Municipio de Envigado, durante el año 2016. Revista Katharsis, N 23, enero-junio 2017, pp. 34-59, Disponible en http://revistas.iue.edu.co/index.php/katharsis 
afianzar el vínculo y a crear espacios en donde se permitan desarrollar nuevos estilos más asertivos para afrontar las dificultades, sin embargo, en esta investigación, lo que se observo es que estos espacios no se generan, puesto que existe una dificultad en cuanto a la manera de comunicarse, y en esa misma vía, no nace la oportunidad de participar de diferentes actividades, ya que no crean una proximidad lo suficientemente estrecha para compartir dichas rutinas.

\section{Afrontamiento de problemas}

En este aspecto se refleja una forma inadecuada de solucionar problemas, relacionada con las dificultades de comunicación, presentes en las familias. La ausencia de la comunicación asertiva al interior de familias no facilita a los menores infractores el aprendizaje de estrategias de solución de conflictos adecuadas (Graña \& Rodríguez, 2010; López-Larrosa et al., 2012). Los testimonios de los adolescentes muestran que en muchas familias no se maneja una forma asertiva de establecer el dialogo, empelando gritos y peleas, lo cual produce un efecto negativo en sus integrantes:

"Para mí los problemas se solucionan dialogando, sin embargo, en mi casa es muy difícil hacerlo así cuando hay alguna dificultad"

"Cuando hay problemas en la casa, siempre gritan y me ponen en el medio, si no me enfurezco no paran, ahí si se concentran en mi para que no me altere"

"Con los problemas mi mamá se desespera, grita, insulta, por eso yo mejor me voy y ella me busca"

"Cuando hay problemas siempre trato de solucionarlos, pero se ha vuelto dificil por el temperamento de mi hijo"

"Los problemas en mi casa se resuelven peleando, discutiendo, yo tengo un problema con eso, no me controlo"

"A veces es mejor quedarse callado porque mi hijo con rabia se descontrola"

$40 \%$ de los adolescentes manifestaron que buscan ayuda para la solución de sus conflictos por fuera del círculo familiar. Esto se refleja, también, en las expresiones de muchos adolescentes participantes que se sienten solos y aislados de sus familiares, enfrentando por si mismos sus problemas o, en el mejor caso, acudiendo a sus amigos u otras personas cercanas (madrina):

"Yo no siento que mi mama me apoye en nada, ella solo está ahí para regañarme, gritarme y pegarme, ella es muy grosera y ya no me quiere ni hablar. Yo siento que mi madrina me quiere más y me apoya a mí"

Citación del artículo: Cardona, E., Martínez, N., Klimenko, O. (2017). Estudio sobre las dinámicas familiares de los adolescentes infractores del programa AIMAR del Municipio de Envigado, durante el año 2016. Revista Katharsis, N 23, enero-junio 2017, pp. 34-59, Disponible en http://revistas.iue.edu.co/index.php/katharsis 
"Yo todos los problemas los he tenido que afrontar sola, a mí no me ayuda nadie"

"Los problemas cada uno tiene que ir mirando como los resuelve, pero con mi mama ya uno no puede esperar nada, ella me utiliza es muy mentirosa y fastidiosa"

"Más fácil busco a los parceros que a la familia cuando tengo problemas"

"Si tengo problemas cuento más con mis amigos que con mi familia, porque ellos no me juzgan"

"Yo creo que puedo enfrentar solo mis problemas"

Lo anterior muestra que algunas familias entrevistadas no cumplen con su función de proveer el apoyo emocional a sus miembros, dejando a los adolescentes vulnerables frente a las problemáticas que viven. En este aspecto autores resaltan la importancia del apoyo familiar, que actúa como un factor protector para los adolescentes en general y sobre todo para los que presentan problemas de delincuencia y/o consumo de sustancias (Gómez \& Bonilla, 2011).

$53 \%$ de los padres manifestaron que no comparten sus problemas con otros familiares, ni amigos. Es notoria la postura de aislamiento familiar en cuanto a la tendencia de no pedir ayuda y no comunicar a los familiares y amigos las dificultades por los cuales están pasando, tratando de resolverlos solos. Lo anterior puede deberse, probablemente, al temor frente al juicio o el rechazo que pueden esperar por parte de los demás.

"Casi no buscamos ayuda de los amigos, somos más bien reservados y siempre en que algo se puede hacer y tratamos de solucionarlo"

"No hablo de mis problemas con mis amigos, en mi casa me ayudan casi siempre"

"En ocasiones nos sentamos a hablar de los problemas que nos pasan y a tratar de solucionarlos"

"Nosotros podemos afrontar solos los problemas, no me gusta comentar con nadie lo que me pasa, menos con mis familiares"

"A mí no me importan los problemas de nadie y no quiero que nadie se meta conmigo"

"A lo largo de los años hemos tratado de enfrentar los problemas juntos solo entre nosotros sin pedirle ayuda a nadie. Al final tenemos que tener claro que la unión hace la fuerza"

Por un lado, es un aspecto que puede ser positivo, tal como expresan algunos adolescentes, ya que sienten que cuentan con el apoyo de sus familiares. Sin embargo, esta tendencia de reservar los problemas solo en el interior de familias y tratar de enfrentarlos solos, puede afectar la capacidad de las familias de enfrentar las problemáticas surgidas, ya

Citación del artículo: Cardona, E., Martínez, N., Klimenko, O. (2017). Estudio sobre las dinámicas familiares de los adolescentes infractores del programa AIMAR del Municipio de Envigado, durante el año 2016. Revista Katharsis, N 23, enero-junio 2017, pp. 34-59, Disponible en http://revistas.iue.edu.co/index.php/katharsis 
que no siempre cuentan con recursos adecuados y suficientes. Los autores resaltan la importancia de emplear las estrategias de afrontamiento de problemas basadas en la búsqueda de ayuda profesional para las familias que atraviesan problemas de consumo y/o delincuencia en los menores (Gómez \& Bonilla, 2011; Graña \& Rodríguez, 2010)

Solo un menor porcentaje de las familias participantes $(20 \%)$ reporta el uso de estrategia basada en la búsqueda de apoyo profesional y/o por parte de las organizaciones comunitarias, como estrategia constructiva, relacionada con la solución de problemas familiares, sobre todo relacionados con el consumo en los adolescentes:

"Si no podemos solucionar los problemas en la casa, trato de buscar ayuda profesional"

"Últimamente con los problemas hemos buscado al psicólogo"

"Yo creo que uno solo no puede resolver nada, si otras personas nos pueden ayudar hay que valorar eso"

"Yo busco en el municipio quien me ayude"

"Cuando surgen dificultades, siempre trato de estar atenta y ayudar a mis hijos, dialogando o buscando ayuda profesional"

También se observa en algunos adolescentes (40\%) una mayor confianza hacia la figura materna como fuente de apoyo para la solución de problemas que se les presentan, en algunas ocasiones, es por tener una familia monoparental y contar solo con la figura materna, $\mathrm{y}$, en otras, porque no sienten confianza en el padre:

"casi no nos pedimos ayuda entre nosotros, pero si es muy difícil lo que tengo que hacer si le pido ayuda a mi mama"

"Si tengo algún problema la única que siempre está conmigo es mi mamá, muy pocas veces mis amigos o algún pariente"

El protagonismo de la madre como figura de confianza y de apoyo ha sido destacado, igualmente, por muchas investigaciones relacionadas con las familias de menores infractores y consumidores de sustancias, quienes resaltan que, independientemente de la composición familiar, la madre emerge como figura de mayor apoyo y protección para los adolescentes, con quien pueden contar con mayor frecuencia para la búsqueda de solución de su problemas en momentos de dificultades (Macías, Amar \& Jiménez, 2005; Gómez \& Bonilla, 2011).

Como se puede observar, a partir de lo anterior, en las familias participantes se evidencian dificultades en el aspecto de solución de problemas familiares, que generan conflictos y peleas al interior de estas, mermando la confianza de adolescentes en los padres, llevándolos a buscar apoyo, en el mejor de los casos, en otras figuras significativas, o en sus pares, que no siempre pueden ser la mejor opción.

Citación del artículo: Cardona, E., Martínez, N., Klimenko, O. (2017). Estudio sobre las dinámicas familiares de los adolescentes infractores del programa AIMAR del Municipio de Envigado, durante el año 2016. Revista Katharsis, N 23, enero-junio 2017, pp. 34-59, Disponible en http://revistas.iue.edu.co/index.php/katharsis 


\section{Satisfacción con la vida}

En cuanto a la satisfacción con su vida $60 \%$ de los adolescentes no definieron su postura, mostrando dudas al respecto de grado de satisfacción con la vida en general.

En sus expresiones se refleja una tendencia de buscar satisfacción en compañía de sus amigos, siendo esto un aspecto común en la edad evolutiva que atraviesan (Ayestarán, 1987; Fuertes, Martínez \& Hernández, 2001).

"En este momento lo único que me tiene feliz es compartir con mis amigos"

Igualmente, se refleja una indecisión y cierto grado de duda en cuanto a su sentir frente a la vida. Lo anterior, puede deberse a las problemáticas que atraviesan en la vida personal y familiar, o a la búsqueda de sentido de vida propia de la edad adolescente (Velasco, 2004; Zubieta \& Delfino, 2010).

"No estoy ni satisfecho ni insatisfecho con mi vida, normal"

"Yo todavía no sé si estoy bien, hay veces me aburro mucho, pero igual que le vamos a hacer"

En cuanto a los padres, solo $40 \%$ respondieron que están satisfechos con la vida, lo cual indica un alto porcentaje de los padres que no se encuentran satisfechos con su vida.

$60 \%$ de los jóvenes no se encontraban ni satisfechos ni insatisfechos con la vida religiosa de su familia, mostrando alta irrelevancia hacia este aspecto de la vida familiar.

En cambio, el 60\% de los padres están bastante satisfechos con la vida religiosa de su familia lo que evidencia que estos últimos manifiestan agrado por el tipo de espiritualidad que sostienen.

En cuanto a los servicios de salud de los cuales dispone la familia solo $33 \%$ de los adolescentes se encuentran satisfechos y un $43 \%$ de los padres se encuentran satisfechos, lo cual muestra un bajo nivel de satisfacen en este aspecto.

En cuanto al barrio y la comunidad en la que viven, un $53 \%$ en los hijos y el $60 \%$ de los padres expresaron su satisfacción, mostrando un nivel relativamente bueno de satisfacción en este aspecto.

En relación a la situación económica de su familia, solo 33\% de los adolescentes y $40 \%$ de los padres expresaron estar bastante satisfechos. Esta insatisfacción, como se puede observar en las expresiones de los padres, se debe, en mayor grado a un alto porcentaje de familias monoparentales con la jefatura femenina, donde la madre es la única que lleva todas las obligaciones del hogar.

"En este momento mi situación está muy difícil me está tocando muy duro con todo a mi sola, yo no quiero seguir así, pero que más le vamos a hacer" 
Igualmente, se percibe en algunos padres, a pesar de la insatisfacción presente, una orientación resiliente de buscar formas de salir adelante y proyectar un mejor futuro para los hijos:

"Tengo para decir frente a esto aunque la situación económica de la familia no es la mejor, no me puedo quejar. En este momento estoy aprendiendo mucho y he tratado de aplicarlo con mi familia. Quiero que mis hijos salgan adelante y estudien para que el día de mañana tengan una mejor vida"

Sobre la satisfacción con el trabajo u ocupación principal el 40\% de los hijos y el 67\% de los padres se encuentran bastante satisfechos.

El ítem de satisfacción con la familia mostro un índice muy bajo en los adolescentes (33\%), lo que demuestra una alta inconformidad con sus familias.

Los adolescentes expresan que buscan un escape en sus amigos o consumo de sustancias, frente a esta insatisfacción familiar:

"En este momento no estoy tan bien, toca esperar a ver qué pasa, me siento inestable y mi relación con mi mamá cada vez está peor, yo prefiero estar en la calle con mis amigos"

"A mí lo del proyecto de vida no me importa. Yo solo quiero a la marihuana y vivir cada día y ya, el resto me da igual. Los pareceros sirven más que la familia."

"Siempre que este con los parceros uno pasa bueno, al menos mejor que con la familia"

Aunque un 53\% de los padres indicaron estar bastante satisfechos con sus familias, el porcentaje restante que no está satisfecho, sigue siendo muy alto. Muchos expresan insatisfacción con el comportamiento y actitud de los hijos y problemas familiares:

"En este momento hay muchos problemas y yo no quiero nada, estoy muy aburrida, esta niña me da mucha lidia, además, en este trabajo casi no se tiene tiempo para nada"

"Estoy un poquito insatisfecha por los problemas familiares y también lo económico"

"si todos estos pelaos son unos mal agradecidos yo que no he hecho sino ayudarlos"

Los datos obtenidos en el estudio muestran, igualmente, problemas a nivel de satisfacción que expresan los adolescentes, en general, con sus familias. Estudios en relación a este aspecto demuestran que los adolescentes consumidores de sustancias muestran un nivel bajo o medio de satisfacción personal con sus familias, lo cual puede estar relacionado con dificultades en comunicación con los padres y conflictos presentes en la familia entre sus miembros (Velásquez \& Pedrão, 2005; Velásquez, Vaiz \& Pedrão, 2009).

Citación del artículo: Cardona, E., Martínez, N., Klimenko, O. (2017). Estudio sobre las dinámicas familiares de los adolescentes infractores del programa AIMAR del Municipio de Envigado, durante el año 2016. Revista Katharsis, N 23, enero-junio 2017, pp. 34-59, Disponible en http://revistas.iue.edu.co/index.php/katharsis 


\section{Conclusiones}

Los datos obtenidos muestran que en las familias participantes se maneja un tipo de comunicación inadecuada e ineficaz que no permite generar una buena cohesión familiar. Lo anterior está asociado, igualmente, a las dificultades en la expresión de emociones entre sus miembros, causando muy poca motivación para promover encuentros que den solución a otros aspectos de su vida en familia.

El ejercicio de autoridad presenta dificultades debido a los desacuerdos entre las figuras paternas, falta de atención hacia los problemáticas de los hijos, lo cual genera que la figura de autoridad se desplaza, a menudo, a otras personas diferentes a los padres. Igualmente, en la mayoría de las familias, la figura de autoridad está representada por la madre, cabeza de familia, a quien se dificulta este rol debido a múltiples ocupaciones de sostenimiento de la familia.

Las rutinas tampoco son adoptadas dentro de las actividades cotidianas debido al mencionado ejercicio inadecuado del dialogo entre unos y otros miembros de las familias. Aunque se observa la presencia de las actividades conjuntas compartidas, sin embrago, estas no evidencias un contacto real afectivo y emocional entre los participantes, limitándose solo al hecho de compartir un espacio físico de la casa o realizar actividades cotidianas.

Los padres entrevistados emplean herramientas inadecuadas para abordar de forma correcta y efectiva los problemas con sus hijos, reforzando de manera negativa sus conductas disfuncionales, a su vez, la ausencia de un vínculo afectivo genera en los jóvenes percepciones de soledad, que los lleva a crear fuera de la familia otro tipo de vínculos que buscan dar respuesta a su necesidad, sin importar que tan adecuados sean estos.

Los resultados del estudio muestran la necesidad de crear estrategias orientadas a la intervención con las familias de los menores infractores y consumidores de sustancias, con el fin de reforzar sus vínculos internos, desarrollar una comunicación asertiva y efectiva entre sus miembros y el empleo de estrategias más funcionales para la solución de conflictos al interior de las familias.

\section{Referencias}

Amezcua, L., García, F., González-Forteza, C., Martínez, N. (2016). Relación entre supervisión parental y conducta antisocial en menores infractores del Estado de Morelos. Salud Mental, 39(1), pp. 11-17. Disponible en http://www.medigraphic.com/pdfs/salmen/sam-2016/sam161c.pdf

Citación del artículo: Cardona, E., Martínez, N., Klimenko, O. (2017). Estudio sobre las dinámicas familiares de los adolescentes infractores del programa AIMAR del Municipio de Envigado, durante el año 2016. Revista Katharsis, N 23, enero-junio 2017, pp. 34-59, Disponible en http://revistas.iue.edu.co/index.php/katharsis 
Arias, W. (2013). Agresión y violencia en la adolescencia: la importancia de la familia. Av.psicol. 21(1), pp. 23-34, Disponible en http://ucsp.edu.pe/imf/wpcontent/uploads/2014/12/Agresionyviolenciaenlaadolescencia1.pdf

Ayestarán, S. (1987). El grupo de pares y el desarrollo psicosocial del adolescente. Disponible en www.raco.cat/index.php/EstudiGral/article/download/43434/56051

Auloss, G. (1983). Finalités individuelles at finalités familiales: Ouvrir des Choix. Revista Thérapie Familiale, Vol. 4. No. 2, pp. 207-219, Disponible en http://www.medhyg.ch/media/upload/Petites_revues/Therapie_familiale/Archives_sc annes/1983-VOL_IV_-N2.pdf

Balarin, M., Cueto, S. (2008). La calidad de la participación de los padres de familia y el rendimiento estudiantil en las escuelas públicas peruanas. GRADE. Disponible en http://www.grade.org.pe/download/pubs/docutrabajo35esp.pdf

Babst, D., et al. (1978). A study of family affinity and substance use. Journal of Drug Education, $\quad$ V. $8(1), \quad$ pp. 29-40, Disponible en http://journals.sagepub.com/doi/pdf/10.2190/DG9F-DBMP-K8YY-8VTR.

Cañavera, M. (1988). Diada Marital disfuncional y farmacodependencia a pasta básica de cocaína. Tesis de bachiller en psicología. Pontificia Universidad Católica del Perú.

Cobos, E. (2008). Adolescencia y familia: revisión de la relación y la comunicación como factores de riesgo o protección. Revista intercontinental de psicología y educación, 10(2), 105-122. Disponible en: http://eds.a.ebscohost.com.bdigital.ces.edu.co:2048/eds/pdfviewer/pdfviewer?sid=2b 0097e8-cc5d-47a4-bb9f-1f0767050184\%40sessionmgr4001\&vid=1\&hid=4205

Chassin, L., Flora, D., King, K. (2004).Trajectories of alcohol and drug use and dependence from adolescence to adulthood: The effects of familial alcoholism and personality. Journal of Abnormal Psychology, 113(4):483-498. DOI:10.1037/0021843X.113.4.483

Domínguez Cuña, A., Rodríguez Machado, E. (2003). La percepción de las relaciones familiares por parte de los adolescentes. Revista galego-portuguesa de psicología e educación, 9, 375-386, Disponible en http://ruc.udc.es/dspace/handle/2183/6950

Fantin, M, García, H. (2011). Factores familiares, su influencia en el consumo de sustancias adictivas. Ajayu, 9(2), Agosto 2011, 193-214, Disponible en http://www.scielo.org.bo/pdf/rap/v9n2/v9n2a1.pdf

Citación del artículo: Cardona, E., Martínez, N., Klimenko, O. (2017). Estudio sobre las dinámicas familiares de los adolescentes infractores del programa AIMAR del Municipio de Envigado, durante el año 2016. Revista Katharsis, N 23, enero-junio 2017, pp. 34-59, Disponible en http://revistas.iue.edu.co/index.php/katharsis 
Fuentes, M., Alarcón, A., García, F., Gracia, E. (2015). Consumo de alcohol, tabaco, cannabis y otras drogas en la adolescencia: efectos de la familia y peligro del barrio. Anales de psicología,31(3), 1000-1007. Disponible en http://scielo.isciii.es/pdf/ap/v31n3/psicologia_adolescencia5.pdf

Fundación W. K. Kellogg (1996). Familia y adolescencia: indicadores de salud. OPS. OMS. Marzo $\quad$ pp. $\quad\left(\begin{array}{llllll}1 & - & 56\end{array}\right)$ Disponible en http://www.codajic.org/sites/www.codajic.org/files/Familia\%20y\%20Adolescencia\% 20\%20Indicadores\%20de\%20Salud\%20Instrumentos.pdf

Fuerte M., Maya M. (2001). Atención a la familia: la atención familiar en situaciones concretas. Anales de sistema sanitario de Navarra, V. 24 (2), pp. 83-92, Disponible en http://recyt.fecyt.es/index.php/ASSN/article/view/5901/4756

Fuertes, A., Martínez, J., Hernández, A. (2001). Relaciones de amistad y competencia en las relaciones con los iguales en la adolescencia. Rev. de Psicol. Gral y Aplic, 54 (3), 531546 ,

Disponible

en https://www.researchgate.net/profile/Jose_Luis_Martinez_Alvarez/publication/28177 474_Relaciones_de_amistad_y_competencia_en_las_relaciones_con_los_iguales_en _la_adolescencia/links/02bfe5148bbfed57b9000000.pdf

Gallego, A. (2011). Recuperación crítica de los conceptos de familia, dinámica familiar y sus características. Revista Virtual Universidad Católica del Norte, No. 35, pp. 236-345, Disponible en http://revistavirtual.ucn.edu.co/

Gaxiola, J., Frías, M. (2008). Consecuencias de la violencia familiar experimentada directa e indirectamente en niños: depresión, ansiedad, conducta antisocial y ejecución académica. Revista mexicana de psicología, N. 25(2), pp. 237-248, Disponible en: <http://www.redalyc.org/articulo.oa?id=243016308004>

González, I. (2000). Reflexiones acerca de la salud familiar. Revista Cubana de Medicina General Integral, 16(5), pp. 508-512, Disponible en http://scielo.sld.cu/scielo.php?script=sci_arttext\&pid=S0864-21252000000500015

Gómez, A., Bonilla, S. (2011). Percepción del menor infractor de su dinámica familiar. Tesis de grado, Facultad de psicología, Corporación Universitaria LaSallista. Disponible en http://repository.lasallista.edu.co/dspace/bitstream/10567/657/1/PERCEPCION_ME NOR_INFRACTOR_DINAMICA_FAMILIAR.pdf

Graña Gómez, J., Rodríguez Biezma, M. (2010). Tratamiento educativo y terapéutico para menores infractores. Facultad de Psicología, Universidad Complutense de Madrid,

Citación del artículo: Cardona, E., Martínez, N., Klimenko, O. (2017). Estudio sobre las dinámicas familiares de los adolescentes infractores del programa AIMAR del Municipio de Envigado, durante el año 2016. Revista Katharsis, N 23, enero-junio 2017, pp. 34-59, Disponible en http://revistas.iue.edu.co/index.php/katharsis 
Disponible en http://www.observatoriodelainfanciadeasturias.es/documentos/f07022012130358.pdf

Gutiérrez Cárdenas, M. (2014). El acompañamiento familiar y el rendimiento académico. Tesis [Licenciadas en educación preescolar] -- Fundación Universitaria Luis Amigó, Disponible en CD-T372 G984 2014 Biblioteca, Fundación Universitaria Luis Amigó

López-Larrosa, S., Sánchez Souto, V., Mendrini, P. (2012). Los adolescentes y el conflicto interparental destructivo: impacto en la percepción del sistema familiar y diferencias según el tipo de familia, la edad y el sexo de los adolescentes. Universitas Psychologica, 11(4), 1255-1262. Disponible en http://revistas.javeriana.edu.co/index.php/revPsycho/article/viewArticle/1255

López, E., Ochoa, G., Olaizola, J. (2005). El rol de la comunicación familiar y del ajuste escolar en la salud mental del adolescente. Salud mental, 28(4), 81, Disponible en: http://www.medigraphic.com/pdfs/salmen/sam-2005/sam054i.pdf

Mosqueda-Díaz, A., Ferriani, M. (2011). Factores protectores y de riesgo familiar, relacionados con fenómeno de drogas, entre adolescentes tempranos de Valparaíso. Chile. Revista Latino-Americana de Enfermagem, 19(spe), pp. 789-795, Disponible en: http://www.scielo.br.bdigital.ces.edu.co:2048/pdf/rlae/v19nspe/17.pdf

Martínez, A., Hernández Cera, M., Arévalo, M., de Jesús, P., Vargas Santillán, M., Lourdes, D. (2014). Funcionamiento familiar de consumidores de sustancias adictivas con y sin conducta delictiva. Liberabit, 20(1), 109-117. Disponible en: http://www.scielo.org.pe/scielo.php?pid=S1729-

48272014000100010\&script=sci_arttext\&tlng=en

Macías, M., Amar, J., Jiménez, M. (2005). Dinámica de las familias de menores con problemas psicosociales: el caso del menor infractor y la menor explotada sexualmente. Revista Latinoamericana de ciencias sociales, niñez y juventud, Vol.3, N.2, pp. 1-24, Disponible en http://www.scielo.org.co/pdf/rlcs/v3n2/v3n2a06.pdf

Medina, N., Carvalho, M. (2010). Factores protectores de las familias para prevenir el consumo de drogas en un municipio de Colombia. Rev. Latino-Am. Enfermagem, 18(Spec): 504-12, Disponible en www.eerp.usp.br/rlae

Navarrete Acuña, L., Ossa, C. (2013). Estilos parentales y calidad de vida familiar en adolescentes con conductas disruptivas. Ciencias psicológicas, 7 (1), pp. 47-56, Disponible en http://www.scielo.edu.uy/pdf/cp/v7n1/v7n1a05.pdf

Citación del artículo: Cardona, E., Martínez, N., Klimenko, O. (2017). Estudio sobre las dinámicas familiares de los adolescentes infractores del programa AIMAR del Municipio de Envigado, durante el año 2016. Revista Katharsis, N 23, enero-junio 2017, pp. 34-59, Disponible en http://revistas.iue.edu.co/index.php/katharsis 
Oliveira, O. de, Eternod, M., López, P. (1999). Familia y género en el análisis demográfico. En B. García, (coord.). Mujer, género y población en México. México: El Colegio de México/ Sociedad Mexicana de Demografía.

Oliva, A., Parra, A., Arranz, E. (2008). Estilos relacionales parentales y ajuste adolescente. Infancia y Aprendizaje, 2008, 31 (1), pp. 93-106, Disponible en http://personal.us.es/oliva/estilos\%20parentales\%20tipologico.pdf

Ortiz, A., Soriano, A., Meza, D., Martínez, R., Galván, J. (2006). Uso de sustancias entre hombres y mujeres, semejanzas y diferencias. Resultados del sistema se reporte de información de drogas. Salud Mental, 29(5), 32-37, Disponible en http://new.medigraphic.com/cgi-bin/resumen.cgi?IDARTICULO=13906

Palacio Valencia, M. (2010). Los tiempos familiares en la sociedad contemporánea: la trayectoria de una configuración. Revista Latinoamericana de estudios familiares, Vol. 2, $\quad$ pp. 9-30, $\quad$ Disponible en http://vip.ucaldas.edu.co/revlatinofamilia/downloads/Rlef2_1.pdf

Parada Navas, J. (2010). La educación familiar en la familia del pasado, presente y futuro. Educatio Siglo XXI, Vol. $28 \quad \mathrm{n}^{\circ}$ 1, pp. 17-40, Disponible en https://www.forofamilia.org/documentos/EDUCACION\%20-

$\% 20 \mathrm{La} \% 20$ educacion\%20familiar\%20en\%20la\%20familia\%20del\%20pasado,\%20pr esente $\% 20 y \% 20$ futuro.pdf

Pons Diez, J. (1998). El modelado familiar y el papel educativo de los padres en la etiología del consumo de alcohol en los adolescentes. Revista Española de Salud Pública, 72(3), pp. 251-266. Disponible en http://scielo.isciii.es/scielo.php?script=sci_arttext\&pid=S1135-57271998000300010

Pichardo, M.C. (2000). Influencia de los estilos educativos de los padres y el clima social familiar en la adolescencia temprana y media. Granada: Universidad de Granada.

Sanz, M., et al. (2004). El conflicto parental y el consumo de drogas en los hijos y las hijas. AEFFA - Asociación para el Estudio del Funcionamiento Familiar, Disponible en http://www.osakidetza.euskadi.eus/contenidos/informacion/publicacions_ovd_otras_i nvesti/es_9061/adjuntos/conflicto_parental.pdf

Torres Velázquez, L. E., Ortega Silva, P., Garrido Garduño, A. \& Reyes Luna, A. G. (2008). Dinámica familiar en familias con hijos e hijas. Revista Intercontinental de Psicología y Educación, 10(2), 31-56, Disponible en http://www.redalyc.org/articulo.oa?id=80212387003

Citación del artículo: Cardona, E., Martínez, N., Klimenko, O. (2017). Estudio sobre las dinámicas familiares de los adolescentes infractores del programa AIMAR del Municipio de Envigado, durante el año 2016. Revista Katharsis, N 23, enero-junio 2017, pp. 34-59, Disponible en http://revistas.iue.edu.co/index.php/katharsis 
Ulate-Gómez, D. (2013). Riesgo biopsicosocial y percepción de la función familiar de las personas adolescentes de sexto grado en la Escuela Jesús Jiménez. Acta méd Costarric, 55(1), $\quad$ pp. $18 \quad-\quad 23 . \quad$ Disponible en: http://www.scielo.sa.cr/pdf/amc/v55n1/art04v55n1.pdf

Valverde, M., Martínez, M., Inchauspe, J. (1991). Intervención familiar y retención de adictos a opiáceos en tratamiento ambulatorio. Adicciones, Vol. 3, No. 4, pp. 24-45.

Vásquez Gonzales, C. (2003). Factores de riesgo de la conducta delictiva en la infancia y adolescencia. En Vázquez González, C. (2003). Delincuencia juvenil. Consideraciones penales y criminologías. Madrid: Colex, pp. 121-168, Disponible en http://www2.uned.es/dpto_pen/delincuencia-juv/documentos/delincuencia/factoresdelincuencia.pdf

Velasco, S. (2004). Búsqueda de sentido de vida en los adolescentes: un modelo centrado en la persona. Tesis de maestría, Universidad Iberoamenricana. Disponible en http://www.bib.uia.mx/tesis/pdf/014425/014425.pdf

Velásquez, D., Pedrão, L. (2005). Satisfacción personal del adolescente adicto a drogas en el ambiente familiar durante la fase de tratamiento en un instituto de salud mental. Rev Latino-am Enfermagem, 13, pp.836-844, Disponible en www.eerp.usp.br/rlae

Velásquez, D., Vaiz, R., Pedrão, L. (2009). Factores de la satisfacción sobre el apoyo familiar del adolescente adicto en tratamiento. Rev enferm Herediana. 2009; 2(1), pp. 11-19, Disponible http://www.upch.edu.pe/faenf/images/pdf/Revistas/2009/enero/ART2_VELASQUEZ .pdf

Viveros, E., Vergara, C. (2014). Familia y dinámica familiar. Medellín: FUNLAM, Disponible en http://www.funlam.edu.co/uploads/fondoeditorial/preliminar/2014/Familia-dinamicafamiliar.pdf

Watzlawick, P. (1974). Teoría de la comunicación. Barcelona: Herder.

Zubieta, E., Delfino, G. (2010). Satisfacción con la vida, bienestar psicológico y bienestar social en estudiantes universitarios de Buenos Aires. Anuario de investigaciones, 17, 277-283. https://www.researchgate.net/publication/262503660_Satisfaccion_con_la_vida_bien estar_psicologico_y_bienestar_social_en_estudiantes_universitarios_de_Buenos_Air es 